\title{
Basic science: (JUNE 2008)
}

1. Akcakanat A, Sahin A, Shaye AN, Velasco MA, Meric-Bernstam F. Comparison of Akt/mTOR signaling in primary breast tumors and matched distant metastases. Cancer 2008; 112: 2352-2358.

2. Apetoh L, Tesniere A, Ghiringhelli F, Kroemer G, Zitvogel $L$. Molecular interactions between dying tumor cells and the innate immune system determine the efficacy of conventional anticancer therapies. Cancer Res 2008; 68: 4026-4030.

3. Bartella V, Cascio S, Fiorio E, Auriemma A, Russo A, Surmacz E. Insulin-dependent leptin expression in breast cancer cells. Cancer Res 2008; 68: 4919-4927.

4. Botlagunta $M$, Vesuna $F$, Mironchik $Y$, Raman $A$, Lisok A, Winnard P, Mukadam S, Van Diest $P$, Chen $\mathrm{JH}$, Farabaugh $\mathrm{P}$, Patel AH, Raman V. Oncogenic role of DDX3 in breast cancer biogenesis. Oncogene 2008; 27: 3912-3922.

5. Cappelletti V, Gariboldi M, De Cecco L, Toffanin S, Reid JF, Lusa L, Bajetta E, Celio L, Greco M, Fabbri A, Pierotti MA, Daidone MG. Patterns and changes in gene expression following neoadjuvant anti-estrogen treatment in estrogen receptor-positive breast cancer. Endocr-Rel Cancer 2008; 15: 439-449.

6. D'Assoro AB, Busby R, Acu ID, Quatraro C, Reinholz MM, Farrugia DJ, Schroeder MA, Allen C, Stivala F, Galanis E, Salisbury JL. Impaired p53 function leads to centrosome amplification, acquired ER $\alpha$ phenotypic heterogeneity and distant metastases in breast cancer MCF-7 xenografts. Oncogene 2008; 27: 3901-3911.

7. Dhar G, Banerjee S, Dhar K, Tawfik O, Mayo MS, VanVeldhuizen PJ, Banerjee SK. Gain of oncogenic function of p53 mutants induces invasive phenotypes in human breast cancer cells by silencing CCN5/WISP-2. Cancer Res 2008; 68: 4580-4587.

8. Grady D, Cauley JA, Geiger MJ, Kornitzer M, Mosca L, Collins P, Wenger NK, Song J,

BCO/843/2008/JW
Mershon J, Barrett-Connor E. Reduced incidence of invasive breast cancer with raloxifene among women at increased coronary risk. J Natl Cancer Inst 2008; 100: 854-861.

9. Hong F, Larrea MD, Doughty C, Kwiatkowski DJ, Squillace R, Slingerland JM. mTOR-raptor binds and activates SGK1 to regulate p27 phosphorylation. Mol Cell 2008; 30: 701-711.

10. Kijima I, Ye J, Glackin C, Chen S. CCAAT/ enhancer binding protein $\delta$ up-regulates aromatase promoters I.3/II in breast cancer epithelial cells. Cancer Res 2008; 68: 4455-4464.

11. Kojima $Y$, Akimoto $K$, Nagashima $Y$, Ishiguro $H$, Shirai S, Chishima T, Ichikawa Y, Ishikawa T, Sasaki T, Kubota Y, Inayama Y, Aoki I, Ohno S, Shimada $\mathrm{H}$. The overexpression and altered localization of the atypical protein kinase $C \lambda / \iota$ in breast cancer correlates with the pathologic type of these tumors. Hum Pathol 2008; 39: 824-831.

12. Lee JY, Jang KS, Shin $D H$, Oh MY, Kim HJ, Kim Y, Kong G. Mel-18 negatively regulates INK4a/ARF-independent cell cycle progression via Akt inactivation in breast cancer. Cancer Res 2008; 68: 4201-4209.

13. Lipton A, Leitzel K, Chaudri-Ross HA, Evans DB, Ali SM, Demers L, Hamer P, Brown-Shimer S, Pierce K, Gaur V, Carney W. Serum TIMP-1 and response to the aromatase inhibitor letrozole versus tamoxifen in metastatic breast cancer. J Clin Oncol 2008; 26: 2653-2658.

14. Masri S, Phung S, Wang X, Wu XW, Yuan YC, Wagman L, Chen S. Genome-wide analysis of aromatase inhibitor-resistant, tamoxifen-resistant, and long-term estrogen-deprived cells reveals a role for estrogen receptor. Cancer Res 2008; 68: 4910-4918.

15. McAllister SS, Gifford AM, Greiner AL, Kelleher SP, Saelzler MP, Ince TA, Reinhardt F, Harris LN, Hylander BL, Repasky EA, Weinberg RA. Systemic endocrine instigation of indolent tumor growth requires osteopontin. Cell 2008; 133: 994-1005.

16. Naderi A, Hughes-Davies L. A functionally significant cross-talk between androgen receptor 
and ErbB2 pathways in estrogen receptor negative breast cancer. Neoplasia 2008; 10: 542-548.

17. Oxmann D, Held-Feindt J, Stark AM, Hattermann $\mathrm{K}$, Yoneda T, Mentlein R. Endoglin expression in metastatic breast cancer cells enhances their invasive phenotype. Oncogene 2008; 27 : 3567-3575.

18. Park CC, Zhang HJ, Yao ES, Park CJ, Bissell MJ. $\beta(1)$ integrin inhibition dramatically enhances radiotherapy efficacy in human breast cancer xenografts. Cancer Res 2008; 68: 4398-4405.

19. Pervin S, Tran AH, Zekavati S, Fulknto JM, Singh R, Chaudhuri G. Increased susceptibility of breast cancer cells to stress mediated inhibition of protein synthesis. Cancer Res 2008; 68: 4862-4874.

20. Rabi T, Banerjee $S$. Novel synthetic triterpenoid methyl 25-hydroxy-3-oxoolean-12-en-28-oate induces apoptosis through JNK and p38 MAPK pathways in human breast adenocarcinoma MCF-7 cells. Mol Carcinog 2008; 47: 415-423.

21. Rakha EA, El-Sheikh SE, Kandil MA, El-Sayed ME, Green AR, Ellis IO. Expression of BRCAI protein in breast cancer and its prognostic significance. Hum Pathol 2008; 39: 857-865.

22. Salnis GJ, Macedo LF, Goloubeva O, Schayowitz A, Brodie AMH. Stopping treatment can reverse acquired resistance to letrozole. Cancer Res 2008; 68: 4518-4524.

23. Shankar E, Sivaprasad U, Basu A. Protein kinase $C \varepsilon$ confers resistance of MCF-7 cells to TRAIL by Akt-dependent activation of $\mathrm{Hdm} 2$ and downregulation of p53. Oncogene 2008; 27: 3957-3966.

24. Stacey SN, Manolescu A, Sulem P, Thorlacius S, Gudjonsson SA, Jonsson GF, Jakobsdottir M, Bergthorsson JT, Gudmundsson J, Aben KK, Strobbe LJ, Swinkels DW, van Engelenburg KCA, Henderson BE, Kolonel LN, Le Marchand L, Millastre E, Andres R, Saez B, Lambea J, Godino J, Polo E, Tres A, Picelli S, Rantala J, Margolin S, Jonsson T, Sigurdsson H, Jonsdottir T, Hrafnkelsson J, Johannsson J, Sveinsson T, Myrdal G, Grimsson HN, Sveinsdottir SG, Alexiusdottir K, Saemundsdottir J, Sigurdsson A, Kostic J, Gudmundsson L, Kristjansson K, Masson G, Fackenthal JD, Adebamowo C, Ogundiran T, Olopade OI, Haiman CA, Lindblom
A, Mayordomo Jl, Kiemeney LA, Gulcher JR, Rafnar T, Thorsteinsdottir U, Johannsson OT, Kong A, Stefansson K. Common variants on chromosome $5 \mathrm{p} 12$ confer susceptibility to estrogen receptor-positive breast cancer. Nat Genet 2008; 40: 703-706.

25. Subtil-Rodriguez A, Millan-Arino L, Quiles I, Ballare $C$, Beato $M$, Jordan A. Progesterone induction of the $11 \beta$-hydroxysteroid dehydrogenase type 2 promoter in breast cancer cells involves coordinated recruitment of STAT5A and progesterone receptor to a distal enhancer and polymerase tracking. Mol Cell Biol 2008; 28: 3830-3849.

26. Taddei I, Deugnier MA, Faraldo MM, Petit V, Bouvard D, Medina D, Fassler R, Thiery JP, Glukhova MA. $\beta 1$ integrin deletion from the basal compartment of the mammary epithelium affects stem cells. Nat Cell Biol 2008; 10: 716-722.

27. Urruticoechea A, Aguilar H, Sole X, Capella G, Martin LA, Dowsett M, Germa-Lluch JR. Preclinical validation of early molecular markers of sensitivity to aromatase inhibitors in a mouse model of post-menopausal hormone-sensitive breast cancer. Breast Cancer Res Treatment 2008; 109: 463-470.

28. Wallach-Dayan SB, Rubinstein AM, Hand C, Breuer R, Naor D. DNA vaccination with CD44 variant isoform reduces mammary tumor local growth and lung metastasis. Mol Cancer Ther 2008; 7: 1615-1623.

29. Zhang M, Behbod F, Atkinson RL, Landis MD, Kittrell F, Edwards D, Medina D, Tsimelzon A, Hilsenbeck S, Green JE, Michalowska AM, Rosen JM. Identification of tumor-initiating cells in a p53-null mouse model of breast cancer. Cancer Res 2008; 68: 4674-4682.

30. Zhang $W H$, Poh A, Fanous AA, Eastman A. DNA damage-induced $S$ phase arrest in human breast cancer depends on Chk1, but G(2) arrest can occur independently of Chk1, Chk2 or MAPKAPK2. Cell Cycle 2008; 7: 1668-1677.

Prepared by $R$ Sutherland Cancer Research Program Garvan Institute of Medical Research Darlinghurst, NSW, Australia 